\title{
Applications of High-Resolution Spatial Discretization Scheme and Jacobian-Free Newton-Krylov Method In Two-Phase Flow Problems
}

\author{
Ling Zou ${ }^{1}$, Haihua Zhao, and Hongbin Zhang \\ Idaho National Laboratory, P.O. Box 1625, Idaho Falls, ID 83415-3870 USA
}

\begin{abstract}
The majority of the existing reactor system analysis codes were developed using low-order numerical schemes in both space and time. In many nuclear thermal-hydraulics applications, it is desirable to use higher-order numerical schemes to reduce numerical errors. High-resolution spatial discretization schemes provide high order spatial accuracy in smooth regions and capture sharp spatial discontinuity without nonphysical spatial oscillations. In this work, we adapted an existing high-resolution spatial discretization scheme on staggered grids in two-phase flow applications. Fully implicit time integration schemes were also implemented to reduce numerical errors from operator-splitting types of time integration schemes. The resulting nonlinear system has been successfully solved using the Jacobian-free Newton-Krylov (JFNK) method. The highresolution spatial discretization and high-order fully implicit time integration numerical schemes were tested and numerically verified for several two-phase test problems, including a two-phase advection problem, a two-phase advection with phase appearance/disappearance problem, and the water faucet problem. Numerical results clearly demonstrated the advantages of using such highresolution spatial and high-order temporal numerical schemes to significantly reduce numerical diffusion and therefore improve accuracy. Our study also demonstrated that the JFNK method is stable and robust in solving two-phase flow problems, even when phase appearance/disappearance exists.
\end{abstract}

Keywords: Jacobian-free Newton-Krylov method, High-resolution spatial discretization scheme, Staggered grid, Implicit scheme

\section{INTRODUCTION}

The majority of the existing reactor system analysis codes, such as RELAP5 [1] and TRAC [2], were originally developed in the mid- to late 1970s. They have played very important roles in the nuclear engineering field by supporting reactor safety analyses as well as design and licensing of new reactors. These codes employed low (first) order numerical methods in both space and time to solve the one-dimensional, two-phase flow equations in reactor systems. The disadvantages of using low-order numerical methods have long been realized in the nuclear thermal-hydraulics field. The classic Implicit Continuous-Fluid Eulerian [3-4] type of operator-splitting method (also known as the semi-implicit method) is commonly used as the time integration scheme. The method introduces a first-order numerical error in time from the operator-splitting process, and it is subject to the material Courant-Friedrichs-Lewy (CFL) stability limit. For spatial discretization, staggered grid mesh combined with the donor cell upwind method is generally used in existing codes for its simplicity, stableness, and ability to preserve monotonicity. However, these schemes are highly diffusive and not always desirable in many applications such as boron solute transport. As new reactor designs emerge, there are new challenges appearing in numerical simulations of reactor systems, such as long transient problems and strongly coupled multi-physics problems. As we aim to improve the numerical accuracy of reactor safety analysis codes, it is important to

${ }^{1}$ Corresponding author, Email: ling.zou@inl.gov 
consider advanced numerical schemes and methods. Our approach includes three aspects. These include: 1) high-resolution spatial discretization scheme in order to improve the spatial accuracy; 2) fully implicit time integration schemes in order to allow large time step and to improve the temporal accuracy; and 3) advanced solving method (such as the Jacobian-free Newton-Krylov (JFNK) method) to efficiently solve the highly nonlinear system.

High-resolution spatial discretization schemes are able to maintain higher-order accuracy in smooth regions while nonphysical spatial oscillations are removed or significantly reduced near discontinuities. However, there have been very few attempts to apply such high-resolution methods in the nuclear thermal-hydraulics field. In addition, most of these works use explicit time integration schemes [5-8], or semi-implicit schemes already implemented in existing system analysis codes [9-11]. For example, Tiselj and Petelin [5] made an attempt to apply a highresolution scheme to solve the two fluid, two-phase flow equations. Special treatments have been done to render the equation system to be hyperbolic in order to use their second-order accurate scheme based on a high-resolution shock capturing method. The explicit time integration method was used. Some of the other existing works are limited to apply high-resolution spatial schemes on scalar variables only, such as boron solute [7-9], or mass/energy equations only [10-11]. A second-order accurate Godunov method was implemented in RELAP5-3D to solve the boron transport equation [9]. However, that scheme is limited to solve the boron transport equation only, and it is lack of thorough tests in system applications according to Ref. [9]. Macián-Juan [7] did an early work to improve the spatial accuracy of boron transport in the system analysis code TRAC using the QUICKEST-ULTIMATE type of flux limiter scheme for one-dimensional flows. Bertolotto et al. [8] has also implemented the same method in the TRACE code to improve the spatial accuracy of the dissolved solute convection equation. This method was developed to use an explicit time integration scheme, and it was applied to the solute equation only. Recently, Wang [10], Wang and his coworkers [11] reported their works on the implementation and assessment of a high-resolution numerical method in TRACE. The high-resolution scheme was applied for the mass and energy equations only, and no treatment was done for the momentum equation. A more restrictive condition on the time-step size than the default CFL limit was also reported in Wang's work [10].

Fully implicit methods are not commonly seen in reactor safety analysis codes, with CATHARE [12-13] as one of the rare exceptions. Attempts to apply Newton's method in solving fully implicit two-phase flow problems can be found in Frepoli's work [14] and Abu Saleem's work [15]. High-resolution spatial scheme was also used in Abu Saleem's work [15], and promising results were obtained. In recent years, the Jacobian-free Newton-Krylov method [16] has gained increasing interest for solving large nonlinear systems. In two-phase flow simulations, Mousseau did several pioneering works [17-19] to use such a method to solve two-phase flow problems implicitly. All Mousseau's works, however, were focused on a first-order upwind spatial discretization scheme. A recent work to apply the JFNK method in solving two-phase flow problems was done by Ashrafizadeh et al. [20]. The spatial discretization scheme is based on a cell centered AUSM+ method, however they were not able to correctly simulate the phase appearance/disappearance problem using the JFNK method.

In this work, it is our objective to investigate the high-resolution spatial discretization scheme on the staggered grid mesh, as well as high-order fully implicit time integration schemes, in the applications of two-phase flow problems. The resulting nonlinear system will be solved by the JFNK method. These numerical schemes could potentially form a foundation to solve the twophase flow problems with better numerical accuracy. In section II, the problem descriptions are given for the simplified two-phase flow problems. In section III, the high-resolution scheme on staggered grid we adapted in this work, along with the time integration schemes and the JFNK 
method will be briefly discussed. In section IV, the applications of high-resolution spatial discretization and high-order implicit time integration schemes on several two-phase flow test problems will be presented. Discussions and conclusions are presented in section V.

\section{PROBLEM DESCRIPTIONS}

For the two-phase flow model, we are particularly interested in the two-fluid, single pressure, two-phase flow equations, which are commonly used in the existing system analysis codes such as RELAP5 [1], TRAC [2], and CATHARE [12]. Since the main purpose is to demonstrate the advantages of using advanced numerical schemes, a simplified version of the two-fluid, single pressure, two-phase flow equations are used in this work. Focusing on the two-phase flow hydrodynamics, and ignoring the mass transfer between the two phases, and wall and interfacial frictions, the six-equation system is further reduced to a four-equation system, including two mass equations and two momentum equations:

$$
\begin{gathered}
\frac{\partial\left(\alpha_{l} \rho_{l}\right)}{\partial t}+\frac{\partial\left[\alpha_{l} \rho_{l} u_{l}\right]}{\partial x}=0 \\
\frac{\partial\left(\alpha_{g} \rho_{g}\right)}{\partial t}+\frac{\partial\left(\alpha_{g} \rho_{g} u_{g}\right)}{\partial x}=0 \\
\frac{\partial u_{l}}{\partial t}+u_{l} \frac{\partial u_{l}}{\partial x}+\frac{1}{\rho_{l}} \frac{\partial p}{\partial x}-g=0 \\
\frac{\partial u_{g}}{\partial t}+u_{g} \frac{\partial u_{g}}{\partial x}+\frac{1}{\rho_{g}} \frac{\partial p}{\partial x}-g=0
\end{gathered}
$$

in which the subscripts $l$ and $g$ denote the liquid phase and the gas phase, respectively. The variables to be solved from this set of equations are $p, \alpha_{g}, u_{l}$, and $u_{g}$, which are pressure, void fraction (volume fraction of the gas phase), liquid phase velocity, and gas phase velocity, respectively. Note that $\alpha_{l}=1-\alpha_{g}$. To close the equation system, linearized equations of state were used for both phases:

$$
\rho_{l}(p)=1000+10^{-7}\left(p-10^{5}\right) ; \rho_{g}(p)=0.5+10^{-6}\left(p-10^{5}\right)
$$

\section{NUMERICAL AND SOLUTION METHODS}

In this section, we will briefly discuss: 1). a high-resolution spatial discretization scheme based on staggered grid mesh arrangement, 2). fully implicit time integration schemes, and 3). the JFNK method in solving the nonlinear equation system.

\subsection{High-resolution staggered grid spatial discretization}

In this work, we adapted a high-resolution spatial discretization scheme on staggered grid mesh, which can be found in the original work done by Stelling and Duinmeijer [21]. The reason that we choose the staggered grid mesh is because it is flexible to handle the two-phase equations in primitive forms, and it is compatible with most existing system analysis codes. The highresolution scheme was obtained by introducing the linear reconstruction of the variable solutions 
and slope limiter into the original first-order upwind method. For the purpose of completeness, the spatial discretization scheme is briefly discussed in this subsection.

For the staggered grid mesh commonly used in existing reactor safety system codes, scalar variables (such as pressure and density) are arranged in cell centers, while vector variables (such as velocity) are arranged on cell edges.

$$
\left.\frac{\partial\left(\alpha_{g} \rho_{g} u_{g}\right)}{\partial x}\right|_{i}=\frac{1}{\Delta x}\left[\left(\alpha_{g} \rho_{g} u_{g}\right)_{i+1 / 2}^{*}-\left(\alpha_{g} \rho_{g} u_{g}\right)_{i-1 / 2}^{*}\right]
$$

and

$$
\left(\alpha_{g} \rho_{g} u_{g}\right)_{i+1 / 2}^{*}=u_{g, i+1 / 2}\left\{\begin{array}{lr}
\alpha_{g, i} \rho_{g, i} & \text { if } \begin{array}{c}
u_{g, i+1 / 2}>0 \\
\alpha_{g, i+1} \rho_{g, i+1}
\end{array} \quad \text { otherwise }
\end{array}\right.
$$

The discretization of the advection term in the momentum equations is done in a similar way,

$$
\left.u_{g} \frac{\partial u_{g}}{\partial x}\right|_{i+1 / 2}=\frac{1}{\Delta x} u_{g, i+1 / 2}\left\{\begin{array}{lrr}
u_{g, i+1 / 2}-u_{g, i-\frac{1}{2}} & \text { if } & u_{g, i+1 / 2}>0 \\
u_{g, i+3 / 2}-u_{g, i+\frac{1}{2}} & \text { otherwise }
\end{array}\right.
$$

The high-resolution version of the discretization is obtained by replacing the donor values with linearly reconstructed cell edge values. Equations (7) and (8) become,

$$
\left(\alpha \rho_{g} u_{g}\right)_{i+1 / 2}^{*}=u_{g, i+1 / 2}\left\{\begin{array}{lr}
\alpha_{i+1 / 2}^{-} \rho_{g, i+1 / 2}^{-} & \text {if } u_{g, i+1 / 2}>0 \\
\alpha_{i+1 / 2}^{+} \rho_{g, i+1 / 2}^{+} & \text {otherwise }
\end{array}\right.
$$

and

$$
\left.u_{g} \frac{\partial u_{g}}{\partial x}\right|_{i+1 / 2}=\frac{1}{\Delta x} u_{g, i+1 / 2}\left\{\begin{array}{lr}
u_{i+1}^{-}-u_{i}^{-} & \text {if } \begin{array}{r}
u_{g, i+1 / 2}>0 \\
u_{i+1}^{+}-u_{i}^{+}
\end{array} \text {otherwise }
\end{array}\right.
$$

in which, the superscripts ' + ' and '-' denote reconstructed values (Figure 1). For more details on the high-resolution method, readers are referred to the original work [21].

\subsection{Time integration schemes}

In this section, the time integration schemes are briefly discussed. Our efforts have been focused on the fully implicit time integration schemes. For the general $\theta$ method, the equation system could be semi-discretized as,

$$
\frac{\boldsymbol{U}^{n+1}-\boldsymbol{U}^{n}}{\Delta t}+\theta\left[\frac{\partial \boldsymbol{f}\left(\boldsymbol{U}^{n+1}\right)}{\partial x}\right]+(1-\theta)\left[\frac{\partial \boldsymbol{f}\left(\boldsymbol{U}^{n}\right)}{\partial x}\right]=0
$$

in which, $\Delta t$ is time step size, and superscripts $n$ and $n+1$ denote the old time step and the current time step, respectively. For the $\theta$ parameter, it takes a value of 1 for the time integration scheme to be the first-order Backward Euler scheme, and 0.5 for the standard Crank-Nicolson scheme. The fully implicit second-order BDF2 scheme is semi-discretized as, 


$$
\frac{\boldsymbol{U}^{n+1}-\frac{4}{3} \boldsymbol{U}^{n}+\frac{1}{3} \boldsymbol{U}^{n-1}}{\Delta t}+\frac{2}{3} \frac{\partial \boldsymbol{f}\left(\boldsymbol{U}^{n+1}\right)}{\partial x}=0
$$

It is noted that neither the standard Crank-Nicolson scheme nor the BDF2 scheme is a strongly stability preserving time integration scheme. However, for most of the applications we are interested in, these methods give satisfactory results, and the unstable behavior can be controlled by reducing the time step.

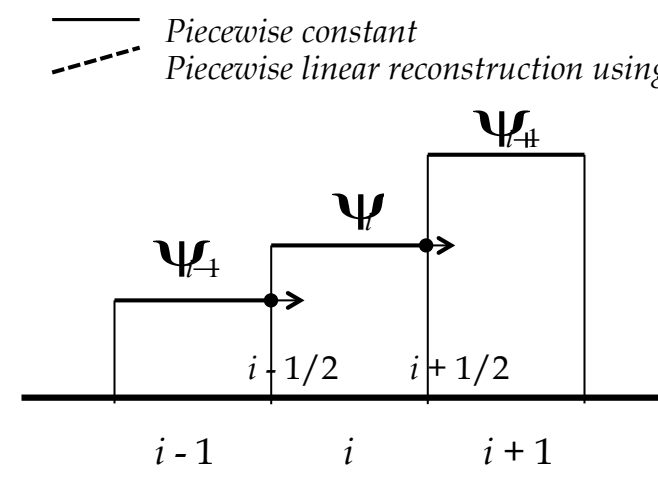

(a) First-order, scalar variables

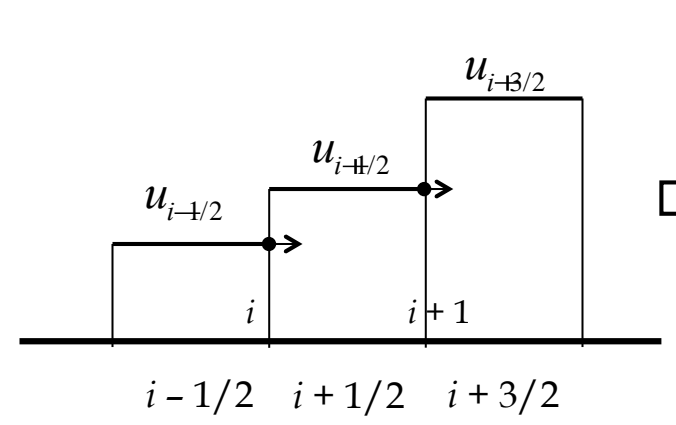

(c) First-order, velocity

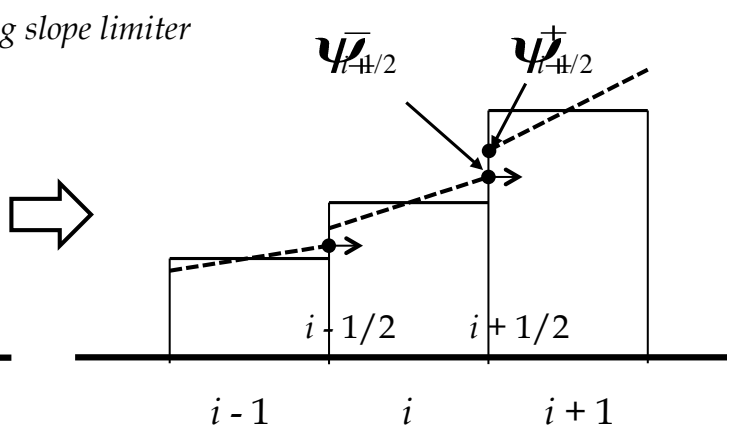

(b) High-resolution, scalar variables

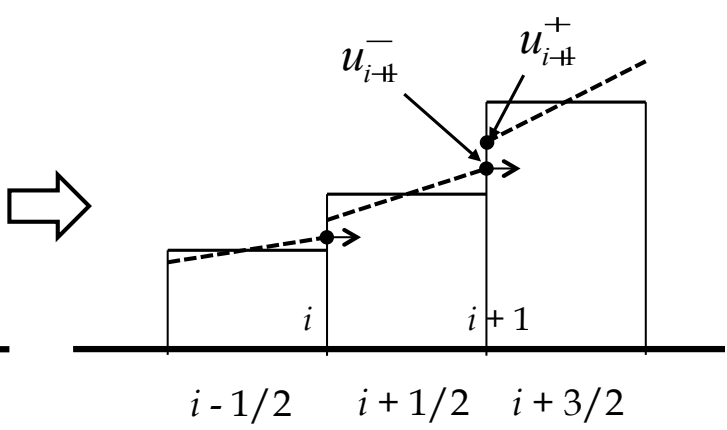

(d) High-resolution, velocity

Figure 1. Schematic illustration of the first-order and high-resolution spatial discretization schemes for the staggered grid mesh. Positive velocities are assumed in this plot. $\psi$ represents scalar properties (such as pressure and void fraction), and $u$ is velocity.

\subsection{Jacobian-free Newton Krylov method}

The resulted discretized nonlinear equation system from the implicit methods requires an iterative method to solve. We are particularly interested in the JFNK method, which has proved its capability in many disciplines and has been used in our previous work in solving two-phase flow problems. In this subsection, the JFNK method to solve nonlinear system is briefly discussed. For a discretized nonlinear system, such as the discretized two-phase equations we are interested in, one solves,

$$
\boldsymbol{F}(\boldsymbol{U})=0
$$


for the unknown vector, $\boldsymbol{U}=\left[p, \alpha, u_{l}, u_{g}\right]^{\mathrm{T}}$ in our two-phase flow problems. The solution to the nonlinear system is obtained by iteratively solving a series of Newton's linear correction equations,

$$
\boldsymbol{J}^{k} \delta \boldsymbol{U}^{k}=-\boldsymbol{F}\left(\boldsymbol{U}^{k}\right)
$$

where, $\boldsymbol{J}^{k}$ is the Jacobian matrix; $\boldsymbol{U}^{k}$ is the $k^{\text {th }}$ nonlinear step solution; and $\delta \boldsymbol{U}^{k}$ is the correction vector. In the JFNK frame, the linear system, equation (14), could be effectively solved with a Krylov's method. In the Krylov's method, only a matrix-vector product is required and thus it does not require the explicit form of the Jacobian matrix. The matrix-vector product can be approximated as,

$$
\boldsymbol{J}^{k} \boldsymbol{v} \approx \frac{\boldsymbol{F}\left(\boldsymbol{U}^{k}+\epsilon \boldsymbol{v}\right)-\boldsymbol{F}\left(\boldsymbol{U}^{k}\right)}{\epsilon}
$$

in which, $\boldsymbol{v}$ is the Krylov vector. After the correction vector, $\delta \boldsymbol{U}^{k}$, is solved from the linear system, the $(k+1)^{\text {th }}$ nonlinear step solution could be updated as,

$$
\boldsymbol{U}^{k+1}=\boldsymbol{U}^{k}+\delta \boldsymbol{U}^{k}
$$

One of the advantages to use the JFNK method is that the explicit formation of the Jacobian matrix could be avoided. The derivation and code implementations of the analytical Jacobian matrix could a cumbersome and error-prone task for two-phase flow system analysis codes, since many thermal-hydraulics correlations have quite complicated forms. In our implementation of the Newton-Krylov method, the scientific computational toolkit PETSc [22] is used to solve the discretized nonlinear fluid equations. It is important to point out that, JFNK method generally needs a good preconditioning scheme to be effective and efficient. There are different ways to precondition the JFNK method, which were reviewed in Ref. [16]. In our work, the preconditioning matrix is obtained by the default finite differencing method provided in the PETSc package.

\section{NUMERICAL TESTS}

In this section, the high-resolution spatial discretization method using the staggered grid mesh will be tested with several benchmark problems interested in the thermal-hydraulics field. These tests include linear advection problems in two-phase flow, and the two-phase water faucet problem. Numerical verification will be done for each test case to demonstrate the second order of spatial accuracy for problems having continuous solutions as well as its excellent capability in capturing sharp spatial discontinuity without oscillations.

\subsection{Linear advection problems}

In nuclear reactor analyses, accurate tracking of scalar quantities, such as boron solute and void fraction distributions in the reactor systems, is essential to perform accurate neutronics and thermal-hydraulics coupling calculations. However, in most existing system analysis codes, due to excessive numerical diffusions from low-order numerical schemes, undesirable less accurate results are often obtained. In this subsection, the linear advection of void fraction problems will be tested with a continuous initial condition, which has a sine distribution in space for the purpose 
of numerical verification. The parameters and initial and boundary conditions for these two tests are summarized in Table $\mathbf{1}$.

Numerical simulations were performed for two numerical configurations: high-resolution spatial scheme + BDF2 (hereafter referred to as high-order methods), and first-order spatial scheme + BDF1 (hereafter referred to as low-order methods). The numerical results of the void fraction distributions are shown in Figure 2, and are compared with the exact solution. From the comparisons, it is clear that, the numerical diffusion is significantly reduced using the higherorder methods. Quantitatively, the $L-1$ error norm of the void fraction is $1.7215 \times 10^{-3}$ when using the high-order methods, an order of magnitude smaller than the $2.8032 \times 10^{-2}$ error norm resulted from using the low-order methods. The $L-1$ error norm follows the definition given in [23], which is proper for hyperbolic equations:

$$
\|E\|_{1}=\Delta x \sum_{i=1}^{N}\left|\tilde{u}_{i}-u_{\text {exact }}\left(x_{i}\right)\right|
$$

where $\Delta x$ is the cell size, $N$ is the total number of cells, $\tilde{u}$ is the numerical solution, and $u_{\text {exact }}$ is the exact solution. A mesh refinement study was also performed to demonstrate the order of accuracy of the high-resolution spatial discretization scheme. A fixed time step size of $10^{-4} \mathrm{~s}$ and total number of time steps of $10^{4}$, and different numbers of cells from 40 to 640 , were used for the mesh refinement study. Table 2 summarized the $L-1$ error norm of the void fraction, from which a second order accuracy was observed, as expected.

Table 1. Parameters, initial and boundary conditions for the linear advection test problem 1

\begin{tabular}{lll}
\hline Parameter & Value & Unit \\
\hline Pipe length $(L)$ & 1 & {$[\mathrm{~m}]$} \\
Gravity $\left(g_{x}\right)$ & 0.0 & {$\left[\mathrm{~m} / \mathrm{s}^{2}\right]$} \\
\hline$u_{l, \text { init }}$ & 1 & {$[\mathrm{~m} / \mathrm{s}]$} \\
$u_{g, \text { init }}$ & 1 & {$[\mathrm{~m} / \mathrm{s}]$} \\
$\alpha_{\text {init }}$ & $0.5+0.2 \sin (2 \pi x / L)$ & {$[-]$} \\
$p_{\text {init }}$ & $10^{5}$ & {$[\mathrm{~Pa}]$} \\
\hline$\alpha_{\text {inlet }}$ & Periodic BC & {$[-]$} \\
$p_{\text {inlet }}$ & $10^{5}$ & {$[\mathrm{~Pa}]$} \\
$p_{\text {outlet }}$ & $10^{5}$ & {$[\mathrm{~Pa}]$} \\
\hline$N_{\text {cell }}$ & $40-640$ & \\
$\Delta t$ & $10^{-4}$ & {$[\mathrm{~s}]$} \\
$N_{\text {timestep }}$ & $10^{4}$ & \\
\hline
\end{tabular}

Table 2. Convergence rate of the linear advection problem

\begin{tabular}{llll}
\hline $\boldsymbol{N}_{\text {cell }}$ & $\boldsymbol{\Delta x}\left(=\boldsymbol{L} / \boldsymbol{N}_{\text {cell }}\right)$ & $\boldsymbol{L}$-1 Norm & Rate \\
\hline 40 & $2.5000 \mathrm{E}-02$ & $6.4528 \mathrm{E}-03$ & - \\
80 & $1.2500 \mathrm{E}-02$ & $1.7215 \mathrm{E}-03$ & 1.91 \\
160 & $6.2500 \mathrm{E}-03$ & $4.3937 \mathrm{E}-04$ & 1.97 \\
320 & $3.1250 \mathrm{E}-03$ & $1.0785 \mathrm{E}-04$ & 2.03 \\
640 & $1.5625 \mathrm{E}-03$ & $2.6283 \mathrm{E}-05$ & 2.04 \\
\hline
\end{tabular}




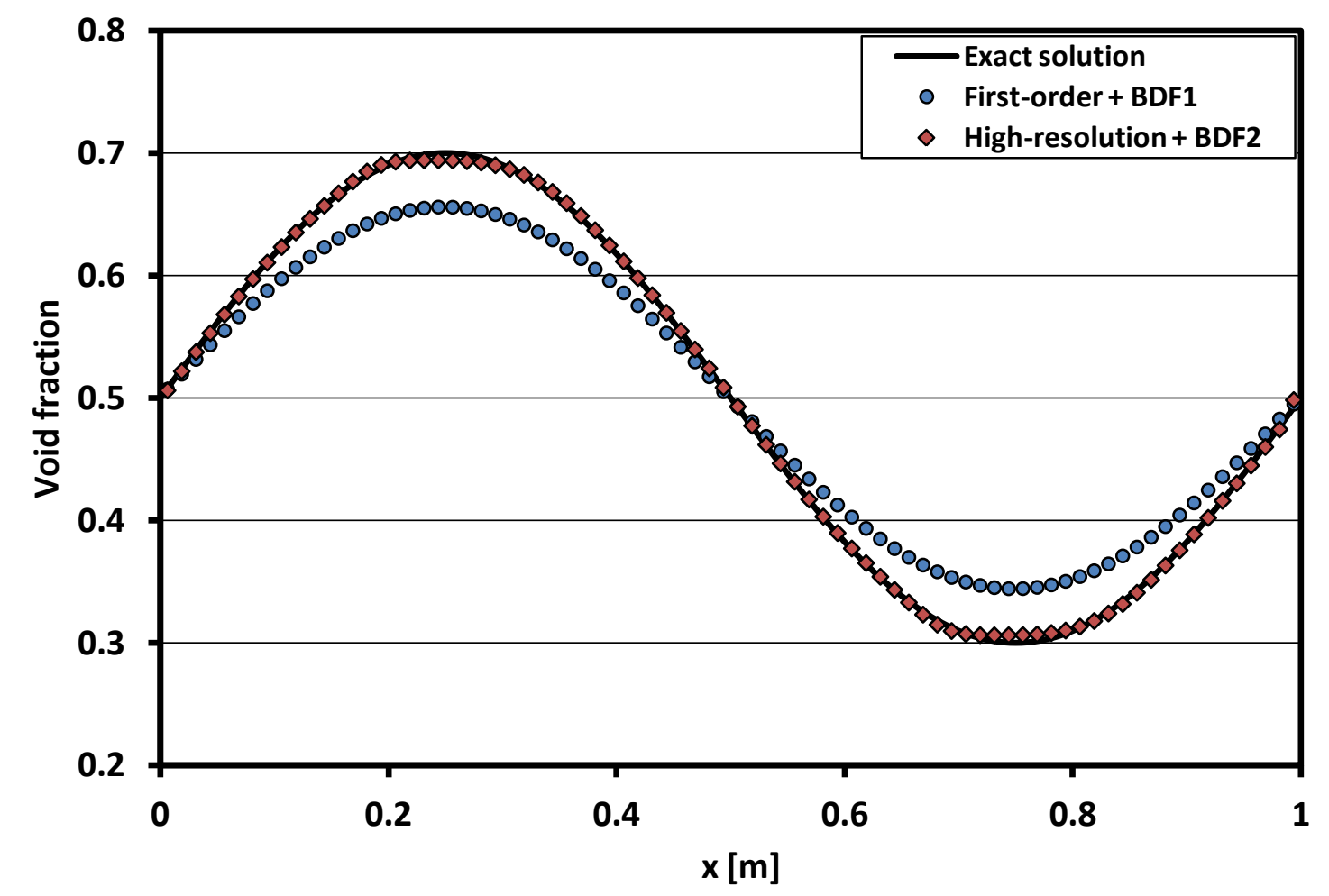

Figure 2. Numerical results of void fraction distribution for the linear advection test problem, with $N_{\text {cell }}=80, \Delta t=10^{-4}$, and $N_{\text {timestep }}=10^{4}$

\subsection{Linear Advection with Phase Appearance/Disappearance}

The phase appearance/disappearance issue presents very serious numerical challenges in the twophase flow simulations. The phase appearance/disappearance in two-phase flow can be caused by advection and/or boiling (condensation). Many existing reactor safety analysis codes use different kinds of treatments for the phase appearance/disappearance problem. However, to our best knowledge, there are no fully satisfactory solutions. A recent work [20], using the JFNK method in solving two-phase flow problems, was not able to simulate such phenomena correctly. The purposes of the linear advection test with phase appearance/disappearance presented in this subsection are two-folds: 1) to test the robustness of the JFNK method in implicitly solving the phase appearance/disappearance phenomenon caused by advection; and 2) to demonstrate the capability of high-resolution spatial discretization scheme and high-order fully implicit time integration scheme in capturing sharp discontinuities. The parameters and initial and boundary conditions for this test are summarized in Tables 3.

In this test, cell numbers 100 and 400 were used. The numerical results of the simulations are shown in Figure 3. It can be observed that, the numerical results from low-order method using 400 cells are only comparable to that from high-order method using 100 cells. Such a comparison between the high-order and low-order methods clearly shows that the high-order methods give much less numerical diffusions. A convergence study was not performed for this test problem, because, in general, the expected order of accuracy cannot be obtained when a discontinuous solution exists (see section 8.7 of [23]). It is also important to point out that, no special treatments were done for conditions where void fraction tends to 0 and 1 . Based on the spatial and temporal schemes used in our work, the JFNK method is able to correctly calculate the void fraction distribution, even when $\alpha=0$ or 1. However, also from Figure 3, it can be observed that the high- 
order method (using 400 cells) gives negative void fraction values (undershoot), due to the fact that the BDF2 scheme is not a strongly stability preserving method. Although the magnitude of undershoot is small (less than $10^{-6}$ ) and the JFNK method could continue well, it may cause serious problems (such as nonphysical value due to negative void fraction value) when realistic closure laws are applied. This issue can be resolved by reducing time step (from $10^{-3}$ to $5 \times 10^{-4}$ ) or by using the more stable BDF1 method, with the results shown in Figure 4. The undershoot behavior of BDF2 indicates that, if high-order fully implicit scheme is desirable, a time step control mechanism in a production code will be necessary.

Table 3. Parameters, initial and boundary conditions for the linear advection with phase appearance/disappearance test problem

\begin{tabular}{|c|c|c|}
\hline Parameter & Value & Unit \\
\hline Pipe length $(L)$ & 1 & {$[\mathrm{~m}]$} \\
\hline Gravity $\left(g_{x}\right)$ & 0.0 & {$\left[\mathrm{~m} / \mathrm{s}^{2}\right]$} \\
\hline$u_{l, \text { init }}$ & 1 & {$[\mathrm{~m} / \mathrm{s}]$} \\
\hline$u_{g, \text { init }}$ & 1 & {$[\mathrm{~m} / \mathrm{s}]$} \\
\hline & if $0.2 \leq x \leq 0.4$ & \\
\hline$\alpha_{\text {init }}$ & otherwise & {$[-]$} \\
\hline$p_{\text {init }}$ & $10^{5}$ & {$[\mathrm{~Pa}]$} \\
\hline$\alpha_{\text {inlet }}$ & periodic B.C. & {$[-]$} \\
\hline$p_{\text {inlet }}$ & $10^{5}$ & [Pa] \\
\hline$p_{\text {outlet }}$ & $10^{5}$ & {$[\mathrm{~Pa}]$} \\
\hline$N_{\text {cell }}$ & $100-400$ & \\
\hline$\Delta t$ & $10^{-3}$ & [s] \\
\hline$N_{\text {timestep }}$ & $10^{2}$ & \\
\hline
\end{tabular}

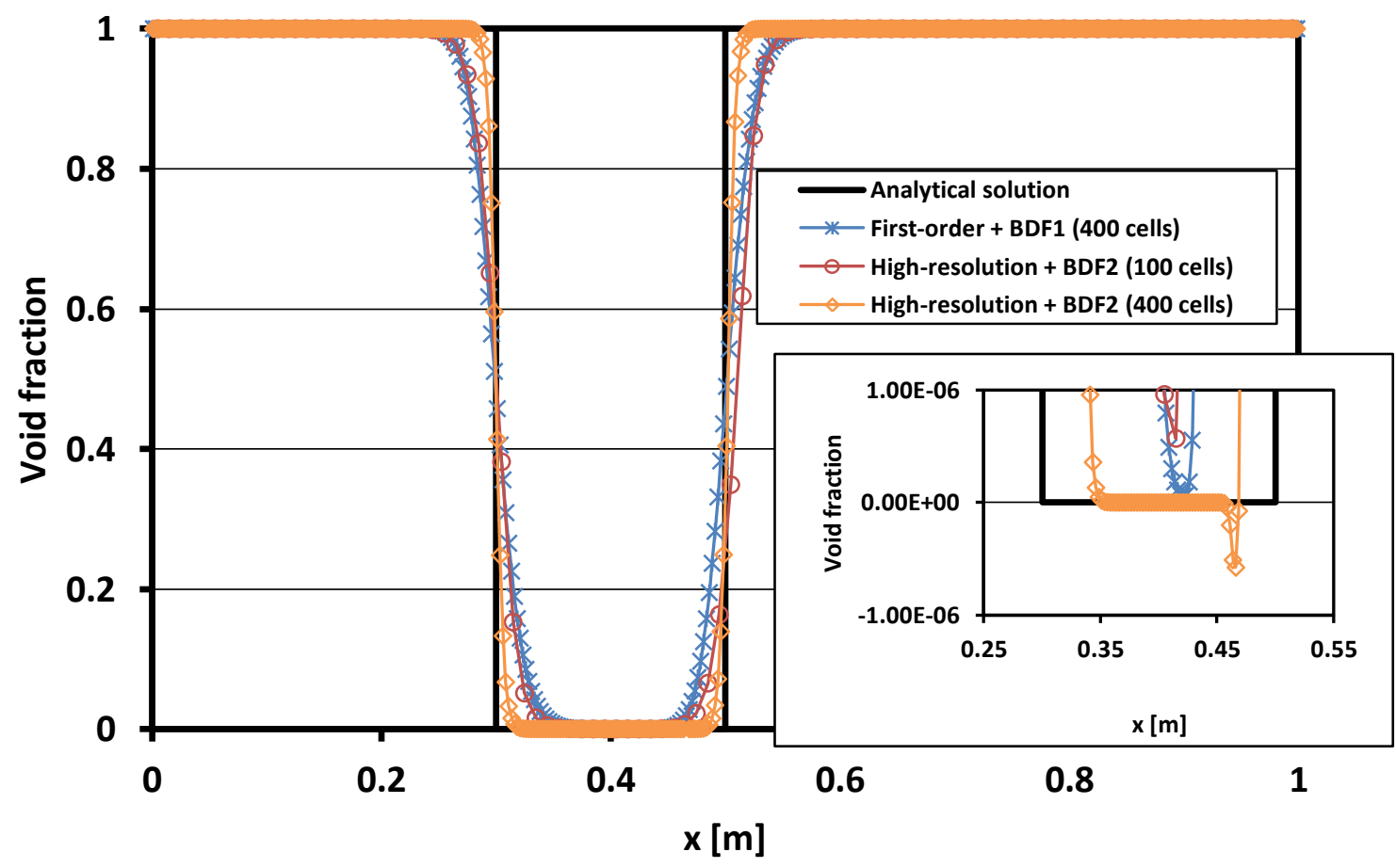

Figure 3. Numerical results of void fraction distribution for the linear advection with phase appearance/disappearance test problem, with $N_{\text {cell }}=100,400, \Delta t=10^{-3}$, and $N_{\text {timestep }}=10^{2}$ 


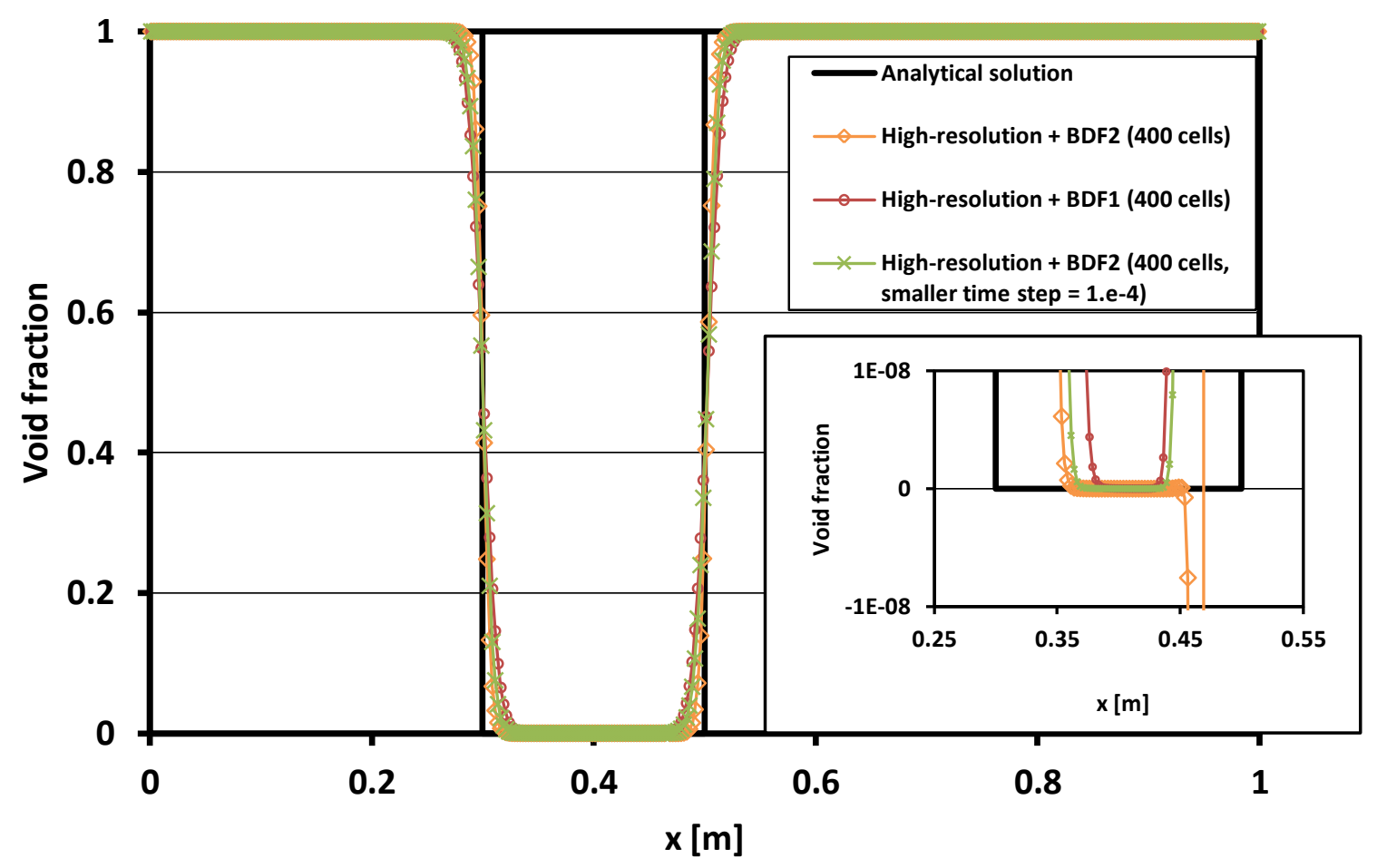

Figure 4. Numerical results of void fraction distribution for the linear advection with phase appearance/disappearance test problem, with $N_{\text {cell }}=400, \Delta t=10^{-3}\left(\right.$ or $\left.5 \times 10^{-4}\right)$ and $N_{\text {timestep }}=$ $10^{2}\left(\right.$ or $\left.2 \times 10^{2}\right)$

\subsection{Water Faucet Problem}

The two-phase water faucet problem is one of the two-phase flow benchmark problems originally proposed by Ransom [24]. This test problem and its analytical solution have been widely used for the purposes of code assessment and benchmark and numerical verifications.

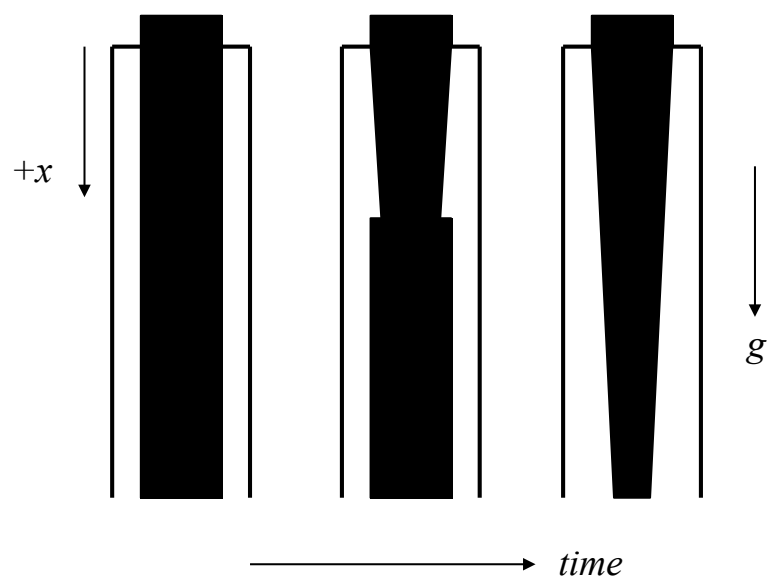

Figure 5. Schematic drawing of the two-phase water faucet problem.

The water faucet problem consists of a vertical pipe of length $L$. Initially, the pipe is filled with a uniform column of liquid moving with velocity $u_{l, \text { init }}$ and an annulus of gas moving with velocity 
$u_{g, \text { init }}$. The initial void fraction (gas phase volume fraction) is $\alpha_{g, \text { init }}$, and the initial pressure is $p_{\text {init }}$. Due to the gravity effect, the liquid column accelerates and becomes thinner with time as it descends. A schematic drawing of the water faucet problem is shown in Figure 5. Analytical solutions with simplifications such as massless gas phase, no wall and interfacial frictions, and no pressure variations in the liquid phase, had been previously obtained for the transient liquid velocity and void fraction distribution [24] (hereafter referred to as the Ransom's solutions):

$$
u_{l}(x, t)=\left\{\begin{array}{cc}
\sqrt{u_{l, \text { init }}^{2}+2 g x} & \text { if } x \leq u_{l, \text { init }} t+g t^{2} / 2 \\
u_{l, \text { init }}+g t & \text { otherwise }
\end{array}\right.
$$

and,

$$
\alpha_{g}(x, t)=\left\{\begin{array}{cc}
1-\frac{\left(1-\alpha_{g, \text { init }}\right) u_{l, \text { init }}}{\sqrt{u_{l, \text { init }}^{2}+2 g x}} & \text { if } x \leq u_{l, \text { init }} t+g t^{2} / 2 \\
\alpha_{g, \text { init }} & \text { otherwise }
\end{array}\right.
$$

Under the steady-state conditions, the Ransom's solutions (Equations (18) and (19)) simply become,

$$
u_{l, S s}(x, t)=\sqrt{u_{l, i n i t}^{2}+2 g x}
$$

and

$$
\alpha_{g, s s}(x, t)=1-\frac{\left(1-\alpha_{g, \text { init }}\right) u_{l, \text { init }}}{\sqrt{u_{l, \text { init }}^{2}+2 g x}}
$$

Numerical simulations were performed using different cell numbers, from 12 to 192, and a fixed time step of $5 \times 10^{-3} \mathrm{~s}$. Figure 6 shows the numerical results of void fraction distribution at $0.5 \mathrm{~s}$ (100 time steps). The results are very similar to those found in the literatures such as the RELAP5 validation report [25], and the work done by Coquel et al. [26] and García Cascales [27]. The numerical result from low-order methods, using 192 cells, is also shown in Figure 6. The result is more diffusive. However, there is no spatial oscillation observed near the discontinuity.

A mesh refinement study was also performed at the steady state condition. It is worth noting that the Ransom's solutions (e.g., equations (18) and (19)) are only approximations to the exact solutions of the two-fluid, single pressure two-phase equations. This solution assumes that there is no pressure variation in the liquid phase and therefore the liquid column admits a "free fall" motion. Nevertheless, these two equations were used as the reference solutions, and the mesh refinement results are summarized in Table 4. As expected, a second order of spatial accuracy could not be obtained. Unfortunately, these approximated analytical solutions have been inappropriately used for numerical verification purposes in many previous works.

In this work, we propose a new set of analytical solutions for the steady state condition. The derivation is simple; however, to the authors' best knowledge, it is not available in literatures. Integrate equation (2) from 0 to $x$ and apply boundary condition $u_{g}(x=0)=0$, to obtain:

$$
u_{g, s s}(x)=0
$$


in which subscript ' $s s$ ' denotes the steady-state condition. Substitute equation (22) into equation (4), and it can be obtained that:

$$
\frac{1}{\rho_{g}} \frac{d p}{d x}-g=0
$$

If $\rho_{g}$ is an integrable function of $\mathrm{p}$, equation (23) could be integrated to get $p(\mathrm{x})$. Here we apply the incompressible approximation, which is proper for the low pressure conditions studied in this work, and the pressure distribution can be obtained as:

$$
p_{s s}(x)=p_{\text {outlet }}-\rho_{g} g(L-x)
$$

The steady-state liquid velocity can be obtained by substituting equation (24) into the liquid phase momentum equation (3):

$$
u_{l, s s}(x)=\sqrt{u_{l, i n i t}^{2}+2 g_{e f f} x}
$$

in which, $g_{\text {eff }}$ is the effective gravity considering the effect of gas phase pressure on the liquid phase. It is defined as,

$$
g_{e f f}=g\left(1-\frac{\rho_{g}}{\rho_{l}}\right)
$$

The void fraction distribution at the steady state can be obtained from the liquid phase mass equation:

$$
\alpha_{g, s s}(x)=1-\frac{\left(1-\alpha_{g, \text { inlet }}\right) u_{l, \text { inlet }}}{u_{l, s s}(x)}
$$

Comparing this set of analytical solutions (Equations (25) and (27)) with Ransom's solutions (Equations (20) and (21)), it can be found that the only difference is that in our analytical solutions the effective gravity replaces the gravity in Ransom's solutions. Performing the same mesh refinement as done previously, and using equations (25) and (27) as the reference solutions, a second-order spatial accuracy could be obtained (Table 5). In addition, the numerical results at the steady state are plotted against the new analytical solutions in Figure $\mathbf{7}$ and Figure 8 with very good agreements. 


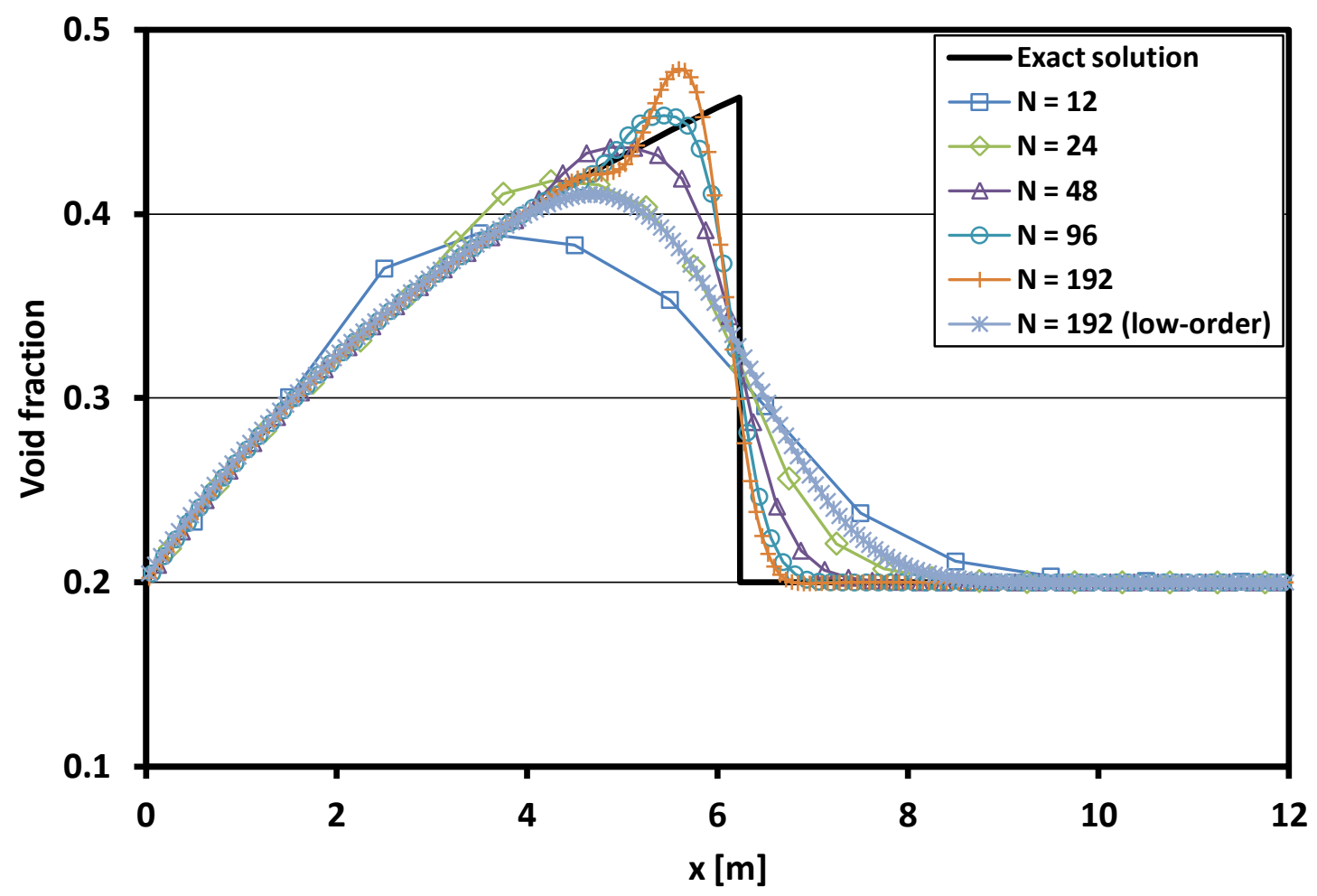

Figure 6. Numerical results of void fraction distribution for the water faucet test problem, with $\Delta t=5 \times 10^{-3}$, and $N_{\text {timestep }}=100$

Table 4. Spatial convergence rate, using the Ransom's solutions as the reference

\begin{tabular}{cccccc}
\hline \multirow{2}{*}{$\boldsymbol{N}_{\text {cell }}$} & $\boldsymbol{\Delta} \boldsymbol{x}=\boldsymbol{L} / \boldsymbol{N}_{\text {cell }}$ & L-1 Norm & $\begin{array}{c}\text { Convergence } \\
\text { Rate }\end{array}$ & L-1 Norm & $\begin{array}{c}\text { Convergence } \\
\text { Rate }\end{array}$ \\
\cline { 3 - 6 } & 1 & $1.9884 \mathrm{E}-02$ & - & $2.7079 \mathrm{E}-01$ & - \\
24 & 0.5 & $6.1237 \mathrm{E}-03$ & 1.70 & $8.9284 \mathrm{E}-02$ & 1.60 \\
48 & 0.25 & $2.1802 \mathrm{E}-03$ & 1.49 & $3.9990 \mathrm{E}-02$ & 1.16 \\
96 & 0.125 & $1.1253 \mathrm{E}-03$ & 0.95 & $2.7052 \mathrm{E}-02$ & 0.56 \\
192 & 0.0625 & $8.5615 \mathrm{E}-04$ & 0.39 & $2.3701 \mathrm{E}-02$ & 0.19 \\
\hline
\end{tabular}

Table 5. Spatial convergence rate, using the new analytical solutions derived in subsection 4.3 as the reference

\begin{tabular}{cccccc}
\hline & \multirow{\Delta}{*}{$\boldsymbol{N}=\boldsymbol{L} / \boldsymbol{N}_{\text {cell }}$} & L-1 Norm & $\begin{array}{c}\text { Convergence } \\
\text { Rate }\end{array}$ & L-1 Norm & $\begin{array}{c}\text { Convergence } \\
\text { Rate }\end{array}$ \\
\cline { 3 - 6 } & 1 & $1.9267 \mathrm{E}-02$ & - & $2.4671 \mathrm{E}-01$ & - \\
24 & 0.5 & $5.4304 \mathrm{E}-03$ & 1.83 & $6.5983 \mathrm{E}-02$ & 1.90 \\
48 & 0.25 & $1.4487 \mathrm{E}-03$ & 1.91 & $1.7085 \mathrm{E}-02$ & 1.95 \\
96 & 0.125 & $3.7463 \mathrm{E}-04$ & 1.95 & $4.3471 \mathrm{E}-03$ & 1.97 \\
192 & 0.0625 & $9.5424 \mathrm{E}-05$ & 1.97 & $1.0953 \mathrm{E}-03$ & 1.99 \\
\hline
\end{tabular}




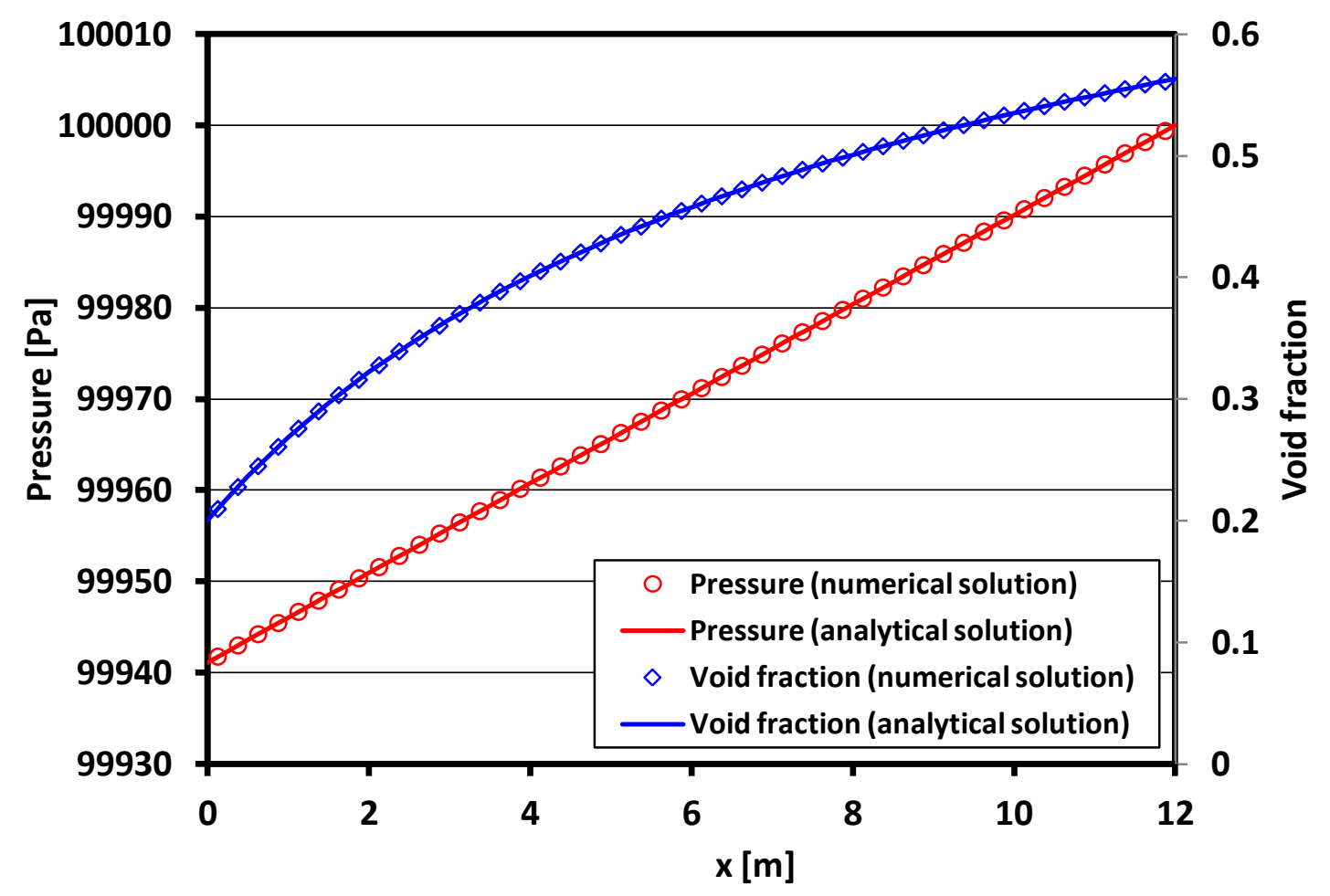

Figure 7. Numerical results of pressure and void fraction with analytical solutions at the steady state.

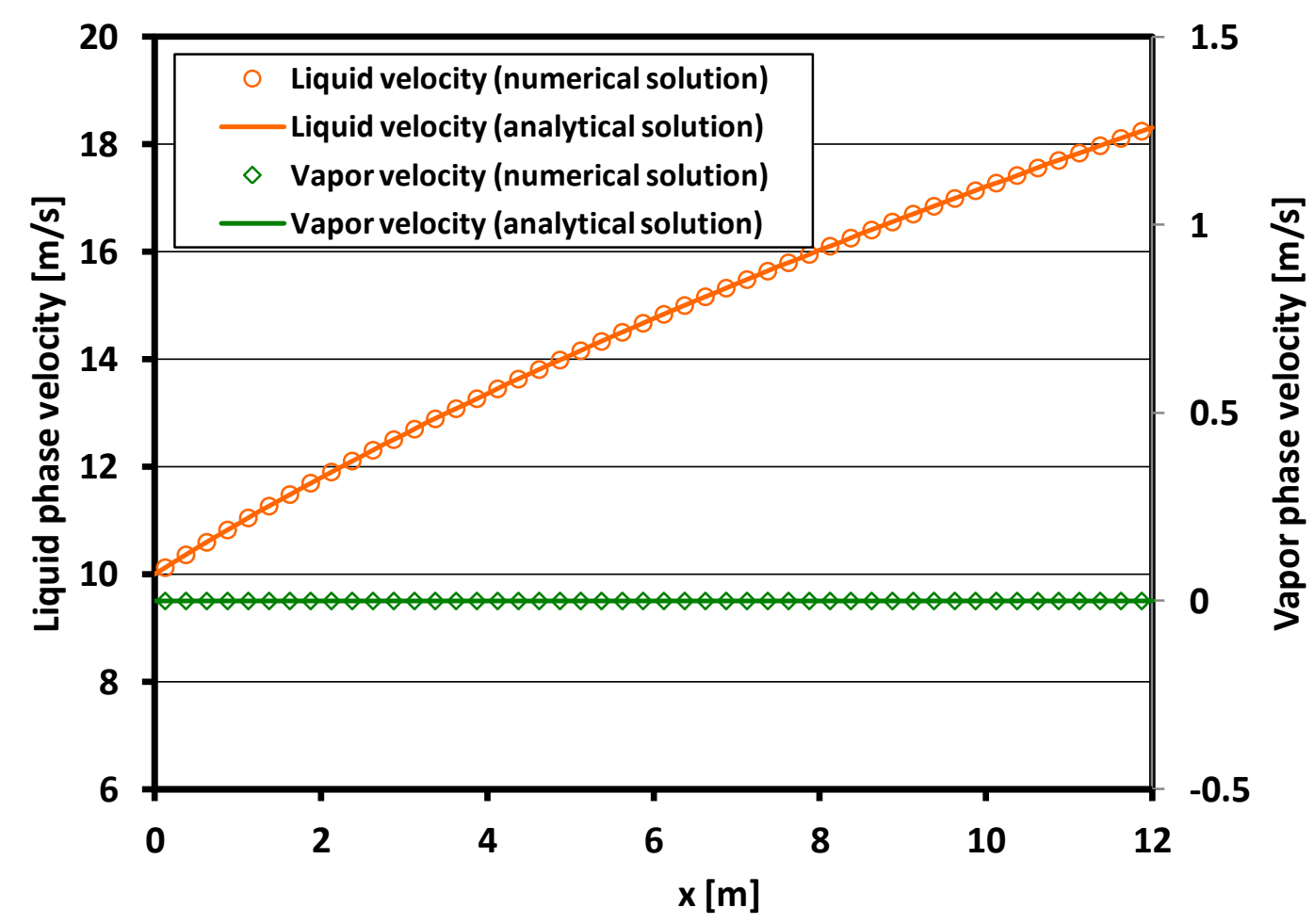

Figure 8. Numerical results of liquid and gas phase velocities with new analytical solutions at the steady state. 


\section{Conclusions}

In this study, high-resolution spatial discretization and high-order fully implicit time integration schemes have been applied to solve one-dimensional two-phase problems. Such a combination is able to significantly reduce numerical errors, which has been demonstrated in several benchmark tests investigated in this work. The discretized nonlinear system was solved with the JFNK method. By using such a method, the cumbersome derivation and error-prone implementation of analytical Jacobian matrix could be avoided. The JFNK method has also demonstrated extremely robust and stable behaviors in solving two-phase flow problem when one phase vanishes or appears. The second-order fully implicit method, BDF2, showed numerical unstable behavior near the sharp discontinuity due to the fact that it is not a strongly stability preserving method. The numerical investigation suggests a time step control mechanism will be necessary if such a high-order fully implicit method is desirable. We have also derived a new set of analytical solutions for the water faucet problem at the steady-state condition. Using these new solutions, a second-order spatial accuracy could be obtained for the high-resolution spatial discretization scheme.

\section{Acknowledgement}

This work is supported by the U.S. Department of Energy, under Department of Energy Idaho Operations Office Contract DE-AC07-05ID14517. Accordingly, the U.S. Government retains a nonexclusive, royalty-free li- cense to publish or reproduce the published form of this contribution, or allow others to do so, for U.S. Government purposes.

\section{References}

1. "RELAP5/MOD3.3 Code Manual Volume I", NUREG/CR- 5535 ed., U.S. Nuclear Regulatory Commission, December, 2001.

2. "TRAC-M/FORTRAN 90 (Version 3.0) Theory Manual”, NUREG/CR-6724 ed., U.S. Nuclear Regulatory Commission, April, 2001.

3. F.H. Harlow and A.A. Amsden, "Numerical Calculation of Almost Incompressible Flow", J. Comp. Phys., Vol. 3, 80-93 (1968).

4. F.H. Harlow and A.A. Amsden, "A Numerical Fluid Dynamics Calculation Method for All Flow Speeds", J. Comp. Phys., Vol. 8, 197-213 (1971).

5. I. Tiselj and S. Petelin, "Modeling of Two-Phase Flow with Second- Order Accurate Scheme", J. Comp. Phys., Vol. 136, 503-521 (1997).

6. S. T. Munkejord, "Analysis of the two-fluid model and the drift-flux model for numerical calculation of two-phase flow", PhD Dissertation, Norwegian University of Science and Technology, 2006.

7. R. Macián-Juan, "A study of high order solute tracking in system codes", Ph.D. Dissertation, Pennsylvania State University (1996).

8. D. Bertolotto, A. Manera, R. Macián-Juan and R. Chawla, "Improvement of the onedimensional dissolved-solute convection equation using the QUICKEST-ULTIMATE algorithm", Nuclear Engineering and Design, 241, 245-256 (2011).

9. "RELAP5-3D Code Manual Volume I: Code Structure, System Mod- els and Solution Methods", INEEL-EXT-98-00834, Revision 4.0, June 2012.

10. D. Wang, "Reduce numerical diffusion in TRACE using the high-resolution numerical method ENO", Transactions of the American Nuclear Society, 107, (2012).

11. D. Wang et al., "Implementation and assessment of high-resolution numerical methods in TRACE”, Nuclear Engineering and Design, 263, 327-341 (2013). 
12. D. Bestion, "The physical closure laws in the CATHARE code", Nucl. Eng. and Des., Vol. 124, 229 (1990).

13. F. Barre, M. Parent, and B. Brun, "Advanced Numerical Methods for Thermalhydraulics", Proceedings of the CSNI Specialist Meeting on Transient Twophase Flow, Aix-en-Provence, France, April 6-8, 1992.

14. C. Frepoli, J. H. Mahaffy, and K. Ohkawa, "Notes on the implementation of a fullyimplicit numerical scheme for a two-phase three-field flow model", Nuclear Engineering and Design, 225, 191-217 (2003).

15. R. A. Abu Saleem and T. Kozlowski, "Development of Accurate and Stable Two-Phase Two-Fluid Model Solver", Proceedings of ICAPP 2014, Charlotte, USA, April 6-9, 2014.

16. D. A. Knoll and D. E. Keyes, "Jacobian-free Newton-Krylov Methods: a Survey of Approaches and Applications", J. Comp. Phys., 193, 357-397 (2004).

17. V. A. Mousseau, "Implicitly Balanced Solution of the Two-phase Flow Equations Coupled to Nonlinear Heat Conduction", J. Comp. Phys., 200, 104-132 (2004).

18. V. A. Mousseau, "Accurate Solution of the Nonlinear Partial Differential Equations from Thermal Hydraulics”, Nuclear Technology, 158, 26-35 (2005).

19. V. A. Mousseau, "A Fully Implicit, Second Order in Time, Simulation of a Nuclear Reactor Core", Proceedings of ICONE-14, International Conference on Nuclear Engineering, Miami, Florida, USA, July 17-20, 2006

20. A. Ashrafizadeh, C.B. Devaud, and N.U. Aydemir, "A Jacobian-free Newton-Krylov Method for Thermalhydraulics Simulations", Int. J. Numer. Meth. Fluids, DOI: 10.1002/fld.3999, 2015.

21. G.S. Stelling and S.P.A. Duinmeijer, "A staggered conservative scheme for every Froude number in rapidly varied shallow water flows", Int. J. Numer. Meth. Fluids, Vol. 43, pp. 1329-1354 (2003).

22. S. Balay et al., "PETSc Users Manual, Reversion 3.4", ANL-95/11 (2013).

23. R. J. LeVeque, "Finite-Volume Methods for Hyperbolic Problems", Cambridge University Press, (2004).

24. Numerical Benchmark Test, volume 2 of Multiphase Science and Technology, Edited by G.F. Hewitt, J.M. Delhaye, and N. Zuber, John Wiley \& Sons Ltd., 1987.

25. "RELAP5/MOD3 Code Manual, Volume 6: Validation of Numerical Techniques in RELAP5/MOD3", NUREG/CR-5535, EGG-2596 October, 1994.

26. F. Coquel et al., A Numerical Method Using Upwind Schemes for the Resolution of Two-Phase Flows, J. Comp. Phys., 136, 272-288 (1997).

27. J. R. García-Cascales, Conservative numerical schemes for unsteady 1D two-phase flow, $\mathrm{PhD}$ thesis, Universidad Politécnica de Valencia, Spain, 2001. 